\title{
Health status, resilience and quality of life of first and fourth year nursing students
}

\author{
Rodrigo Marques da Silva*1, Ana Lucia Siqueira Costa $^{2}$, Fernanda Carneiro Mussi ${ }^{3}$, Fernanda Michelle Santos e Silva ${ }^{3}$, \\ Keila Cristina Félis ${ }^{4}$, Victor Cauê Lopes ${ }^{5}$, Cristilene Akiko Kimura ${ }^{1}$ \\ ${ }^{1}$ Nursing Department, Faculdade de Ciências e Educação Sena Aires, Valparaíso de Goiás, Goiás, Brazil \\ ${ }^{2}$ Nursing Department, Universidade de Sao Paulo, Sao Paulo, Brazil \\ ${ }^{3}$ Nursing Department, Universidade Federal da Bahia, Salvador, Bahia, Brazil \\ ${ }^{4}$ Nursing Departament, Centro Universitário de Goiatuba. Goiatuba, Goiás, Brazil \\ ${ }^{5}$ Nursing Departament, Faculdade do Vale do Juruena. Juína, Mato Grosso, Brazil
}

Received: August 15, 2019

DOI: $10.5430 /$ jnep.v10n2p1
Accepted: September 19, $2019 \quad$ Online Published: October 22, 2019

URL: https://doi.org/10.5430/jnep.v10n2p1

\begin{abstract}
Objective: To compare the health status (stress, depressive symptoms and sleep quality), the resilience and quality of life in first and fourth year nursing students.

Methods: This is a cross-sectional research conducted in 2016 with 86 students enrolled in first and fourth years of the nursing degree. We applied the instrument for Assessment of Stress in Nursing Students, the Center for Epidemiologic Studies Depression Scale, Pittsburg Sleep Quality Index, Wagnild and Young's Resilience Scale; and the WHOQOL-BREF. ANOVA (Test F) was applied for data analysis.

Results and conclusions: A total of 49 first-year and 37 fourth-year students were sampled for this study. Fourth- year nursing students showed higher levels of stress, lower intensity of depressive symptoms and higher quality of life and resilience levels. The poor sleep quality was prevalent in both groups. Conclusion: although the nursing education potentially contributes for students' sickness, the experiences lived in this period may strength the resilience skills.

Conclusions: Video indexing and retrieval are accomplished by using hashing and $k$-d tree methods, while visual signatures containing color, shape and texture information are estimated for the key-frames, by using image and frequency domain techniques. Experimental results with the dataset of a multimedia information system especially developed for managing television broadcast archives demonstrate that our approach works efficiently, retrieving videos in 0.16 seconds on average and achieving recall, precision and $\mathrm{F} 1$ measure values, as high as $0.76,0.97$ and 0.86 respectively.
\end{abstract}

Key Words: Nursing, Nursing students, Mental health, Factor analysis, Statistical

\section{INTRODUCTION}

A set of aspects typical from lifestyle in big cities may affect the individuals' daily, with impact on their health status. It includes the inefficient public transportation, violence, long distances and time spent daily from home to work or college. ${ }^{[1]}$ Also, university students experience specific situations from college setting, such as: taking tests, overloading academic tasks, transition from the pre-university phase to the academic environment, diagnosis and treatment of patients, possibility of error in healthcare, equipment handling and lack of professional knowledge and skills. ${ }^{[1,2]}$

Several stressors were frequent reported for researchers in Brazil and other countries, such as: relation with friends,

\footnotetext{
* Correspondence: Rodrigo Marques da Silva; Email: marques-sm@ @otmail.com; Address: Nursing Department, Nursing Department, Faculdade de Ciências e Educação Sena Aires, Valparaíso de Goiás, Goiás, Brazil. 
faculties and healthcare professionals; difficulty to handle with emotions; low self-confidence; lack of economic resources; and difficult in time management for accomplish the academic demands and conciliate them with personal and social life. ${ }^{[2,3]}$

Specifically in the nursing area, evidences show that this is the most stressful profession and that nursing students present higher levels of stress when compared with other health courses. It may occur due to the personal contact with patients for a longer period of time during the course, what implies frequent contact with death, suffering, disease and barriers faced for health professionals daily. ${ }^{[4]}$

In this sense, the adaptive resources of nursing students may be overloaded due to the academic situations experienced, what may cause stress during the education process. Consequently, these individuals may experience negative outcome to their health, including depressive symptoms, poor sleep quality and reduced quality of life.

Stress in human beings- according the interactionist modelis defined as any stimulus from internal or external environment and that taxes or exceeds the adaptation resources of an individual or social system. ${ }^{[5]}$ The stress occurrence was assessed in 130 nursing students from South Brazil, being that $9.23 \%$ of the sample presented high stress levels and $67.69 \%$ moderate stress levels. ${ }^{[6]}$

Depressive symptoms may lead to suffering and affect individuals in social and academic aspects, what may cause the depressive disorder. ${ }^{[7]}$ This symptomatology assessment is based on affective and behavioral components, such as: depressed mood, feelings of guilty and useless; feelings of lack of support and hopeless; psychomotor delay; loss of appetite; and changes in sleep patterns. ${ }^{[8]}$ In a research conducted with Chinese high school students, $6.4 \%$ had depressive symptoms. ${ }^{[9]}$ In addition, few researchers refer depressive symptoms as predictor of poor sleep quality, fact that was confirmed in investigation performed with 3186 Chinese students-those students with depressive symptoms had 2.47 times more chance to show changes in sleep quality. ${ }^{[9]}$

The sleep quality also may negatively impact on nursing students' health, once the lack of a restful sleep contributes significantly for errors in healthcare, even during the academic phase. ${ }^{[10]}$ The occurrence of poor sleep quality was found in $78.81 \%$ of nursing students from Sao Paulo, ${ }^{[1]}$ being that $23.2 \%$ of the sample showed sleep disturbances once or twice a week. Investigation that took place in India with 750 nursing students reported that students with better sleep quality has less insomnia and depression and more capacity to study for longer time a day. ${ }^{[10]}$ Previous studies reported that nursing students have been experienced academic stress, poor sleep quality and depressive symptoms, events that may negative impact on their quality of life. This is defined as the individual's understanding of their own perception of life from the culture and values in which they are inserted, as well as their relationship with the goals, expectations, pattern and concerns. ${ }^{[11]}$ This relation between health changes and quality of life was reported for a investigation with 1074 university students, where those diagnosed with chronic insomnia had poorer quality of life. ${ }^{[12]}$ In a study with 346 Korean nursing students, we observed that depressive symptoms decreased significantly the students' quality of life. ${ }^{[4]}$

Even face a social and academic setting that increase the risk of illnesses, few person may experience reduced stress levels and, thus, lower chances of suffering from its negative outcomes. ${ }^{[13]}$ This ability of an individual recovering from adversities and positively adapting to a life situations is defined as resilience. ${ }^{[13]}$ Resiliency involves emotional, sociocultural and cognitive aspects that interact to each other and allow to cope with stressors, to strength and transform individuals face the adverse situations and stress experienced. ${ }^{[3,13]}$ In an investigation conducted with 1,538 Chinese nursing students, finding showed that last-year students were more stressed than those from other degree's stages, but the more resilient students showed lower stress levels. ${ }^{[3]}$

After starting college, students are exposed to stressful situations that may affect their physical and mental health, including outcomes as depressive symptoms, poor sleep quality and reduced quality of life. On other hand, resilience may work as a preventive strategy against stress and its negative impact on health. However, most of the mentioned studies have cross-sectional design ${ }^{[1,12]}$ and few of them compared the health status of nursing students across the years of academic education. ${ }^{[3]}$

In this sense, we aimed to compare the health status (stress, depressive symptoms and sleep quality), the resilience and quality of life in first and fourth year nursing students. As research hypothesis, we defined: nursing students show changes in their health status across the degree, being that: fourth-year students has higher stress levels and depressive symptoms; poorer sleep quality and quality of life; as well as higher resilience levels, when compared with entrant students.

\section{METHOD}

This is a cross-sectional, prospective and quantitative research conducted with nursing degree students. The study population was composed for all first and fourth-year nursing students enrolled in one state university located in the state 
of Sao Paulo.

We included students regularly enrolled in the first or fourth years of the degree and aged over 18 years. Those students in exchange during the data gathering were excluded.

The data was gathered in March 2016 trough the following instruments: Instrument for Assessment of Stress in Nursing Students(ASNS), Center for Epidemiologic Studies Depression Scale(CES-D), Pittsburg Sleep Quality Index (PSQI), Wagnild \& Young's Resiliency Scale; and Instrument for Quality of Life Assessment (WHOQOL-BREF). We took permissions from the originators of all instruments by e-mail before the ethical committee approval. These instruments were applied in classrooms after faculties' previous authorization. A date for devolution was scheduled with students after the exposition of study aims and instruments delivery. Resiliency is a new concept in human beings and its correlated factors remains unclear in literature. Any event from 2016 have changed the theoretical framework or empirical knowledge on resiliency. So, these research findings are not outdated.

The Instrument for Assessment of Stress in Nursing Students(ASNS) was proposed by Costa and Polak in 2009 ${ }^{[2]}$ and is composed for 30 items distributed in six domains, as follows:

Realization of Practical Activities (RAP); Environment (AMB); Professional Communication (CP); Time Management (GT); Professional Training (FP); and Theoretical Activity (AT). These items are presented in Likert type scale with four points: zero (0), applied when the student did not experience stress at the situation depicted in the item; one (1), when stress level is regarded as low by the student in the presented situation; two (2), when the situation presents a moderate stress level; and three (3), when the student perceives a high stress level in the situation. ${ }^{[2]}$ To identify stress intensity in each ASNS's factor, we applied risk quartiles as suggested for the instrument's authors. ${ }^{[2]}$ To classify the general stress level in each student, the scores (from 0 to 3 ) attributed to the 30 items were summed and, then, compared between first and fourth year students.

The CES-D was validated in college students for Filho and Teixeira in 2011. ${ }^{[7]}$ This instrument aims to assess the depressive symptomatology experienced one week before its application. The CES-D contains 20 item scales about humor, somatic symptoms, social interactions and motor functioning. They are organized in four subscales, as follows: Depression (Items 3,6,9,10,14,17 and 18), Interpersonal (Items 15 and 19), Positive Affection (Items 4,8,12 and16) e Somatic/Initiative (Items 1,2,5,7,11,13 and 20). These items are presented in a four-point Likert scale, where: 0- rarely(less than one day); 1-For little time (1-2 days); 2- For moderate time(3-4 days); and 3- for most of time(5-7 days). ${ }^{[7]}$

The Pittsburgh Sleep Quality Index (PSQI), after being validated for Brazilian context, was adapted and applied in university Brazilian students from different places. ${ }^{[9]}$ The PSQI is composed for 10 questions that are arranged in seven components: Subjective sleep quality (Question 6); Sleep onset latency (Question 2 and 5a); Sleep duration (Question 4); Habitual sleep efficiency (Questions 1, 3 and 4); Sleep disturbances (Questions 5b to 5j); Use of hypnotic-sedative medication (Question 7); presence of daytime sleepiness and

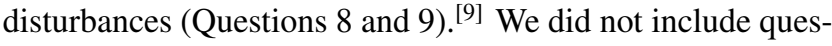
tion 10 because is it requires a roommate for its analysis (Bertolazi, 2008). ${ }^{[9]}$ A global score is generated by summing the components' scores- that ranges from 0 to 3 . The sum that ranges from 0 to 21 points, being that the higher score, the worse is the sleep quality. Scores over 5 points indicate poor sleep quality in the sample assessed. ${ }^{[9]}$

The Resilience Scale, developed for Wagnild and Young in 1993, with adult women. ${ }^{[13]}$ was adapted and translated to the Brazilian population in 2005 , what was done using the public high school students as sample. ${ }^{[14]}$ This instrument measures persons' positive psychosocial adaptation to notable adverse situations in life. It has 25 items presented in a seven-point Likert scale, ranging from 1 (totally disagree) to 7 (totally agree). These items are distributed in 3 factors: resolution of actions and values (Items 1, 2, 6, 8, 10, 12, 14, 16, 18, 19, 21, 23, 24 and 25), independency and determination (Items 5, 7, $9,11,13$ and 22) and self-confidence and ability of adapting to situations (Items 3, 4, 15, 17 and 20). ${ }^{[14]}$ These factors correspond to the attributes that support coping with life issues, including the competency in social relations, the capacity for solving problems, the conquest of autonomy and the purpose of life and future ${ }^{[14]}$ Instrument's score ranges from 25 to 175 points, being that higher scores indicate higher levels of individual's resiliency.

The WHOQOL-BRE was validated for the Brazilian context in $2000^{[15]}$ and is a generic instrument for measuring Quality of Life (QoL).It is composed for 26 items, as follows: two open questions and 24 items described in a five-point Likert scale (from 1 to 5). These 24 items are organized in four domains that approach the individuals' perception of their QoL: Physical (Items 3, 4, 10, 15, 16, 17 and 18), Psychological (Items 5,6, 7, 11, 19 and 26), Social Relations (Items 20, 21 and 22) and Environment (Items 8, 9, 12, 13, 14, 23, 24 and $25) .{ }^{[15]}$ The open questions are assessed separately. Question 1 denotes the individuals' perception about their QoL; and the Question 2 evaluates the respondents' satisfaction with 
their health status. ${ }^{[15]}$

Before the instrument analysis, the scale of items 3, 4 and 26 needed to be inversed, as follows: $1=5 ; 2=4 ; 3=3 ; 4=2 ; 5$ $=1$. Then, the mean for each domain is calculated by the sum of punctuations signed in each item divided for the number of items in the referred domain. ${ }^{[15]}$ The mean of each domain was multiplied by 4 to ensure that the WHOQOL-BREF and WHOQOL-100 scores are comparable. For analyzing the general QoL, we calculated the mean of individual's responses to all items. Higher QoL domains and overall reflects higher respondent's QoL. ${ }^{[15]}$

The Statistical Package for Social Sciences(SPSS) (version 10.0) was used for data analysis. Quantitative variables are presented in descriptive measures: minimum and maximum values, mean, standard deviation. Qualitative variables were assessed in absolute and relative frequencies. For comparing the scores of stress, depressive symptoms, sleep quality, resiliency and quality of life between first and fourth year students, the ANOVA model (Test F) was applied. The Pearson's correlation test was used to assess the association among all constructs. $P$ values $<.05$ were considered statistically significant. Instrument reliability was determined by Cronbach's Alpha-0,7 or above were considered as adequate values. ${ }^{[16]}$

The Research Ethical Committee approved the Project (Protocol n. no1.363.890) in November 11th, 2015. In order to meet the requirements of Health National Council Resolution 466/12, the Free and Informed Consent Term (FICT) were reviewed by the participants and signed (In two copies) with the protocol to all individuals who agreed to participate of the study.

\section{RESUlts}

In March, 86 students were enrolled in the first year and 65 in the fourth year of nursing degree, totalizing 151 students as initial population. However, 49 first-year students and 37 fourth-years students returned the filled protocols, what resulted in an access population of 86 nursing students.

The Alpha values found for the ASNS (30 items) was of 0.873 , for Resiliency Scale (25 items) was of 0.822 ; for WHOQOL-BREF (26 items) was of 0.799 and for the CESD (20 items) was of 0.742 . Thus, once values were over 0.7 , these instruments may be considered reliable to be applied in nursing students. The Table 1 shows the comparison of stress levels, depressive symptoms, sleep quality, resiliency and quality of life between first and fourth-year nursing students.

Table 1. Comparison of stress levels, depressive symptoms, sleep quality, resiliency and quality of life between first and fourth year nursing students. Sao Paulo, 2016.

\begin{tabular}{llll}
\hline \multirow{2}{*}{ Variables } & $\mathbf{1}^{\text {st }}$ year $(\mathbf{n}=\mathbf{4 9})$ & 4th year $(\mathbf{n}=\mathbf{3 7})$ & $\boldsymbol{p}$ value \\
\cline { 2 - 3 } & Mean $(\mathbf{S D})$ & Mean $(\mathbf{S D})$ & $<.001^{*}$ \\
\hline Academic Stress & $46.75(10.7)$ & $61.29(10.14)$ & .420 \\
Sleep Quality & $9.77(2.57)$ & $10.24(2.74)$ & $.027^{*}$ \\
Depressive Symptoms & $43.42(7.93)$ & $39.72(6.99)$ & $<.001^{*}$ \\
Resiliency & $116(16.25)$ & $134(13.61)$ & $.002^{*}$ \\
Quality of Life - Total Score & $3.02(0.38)$ & $3.27(0.32)$ & $<.001^{*}$ \\
Quality of Life - Physical & $2.65(0.37)$ & $3.19(0.41)$ & $<.001^{*}$ \\
Quality of Life - Psychological & $3.18(0.56)$ & $3.67(0.57)$ & .050 \\
Quality of Life - Social Relation & $3.36(0.91)$ & $3.71(0.69)$ & .714 \\
Quality of Life - Environment & $3.04(0.45)$ & $3.08(0.48)$ & \\
\hline
\end{tabular}

*Statically significant difference

The academic stress levels were higher in fourth year students $(p<.001)$. On other hand, these students' group showed lower intensity of depressive symptoms and better quality of life $(p=.002)$ when compared to the first year students. In the domains evaluation, the quality of life in physical and psychological domains were higher in fourth year students. Also, no significant difference was found in sleep quality between the groups, demonstrating that sleep quality is poor throughout the course (Score $>5$ ). Although the stress level be higher in fourth years students, these individuals are more resilient than incoming students $(p<.001)$. In Figure 1 , we present the comparison of means for each Resiliency Scale's domain in first and fourth year nursing students.

Fourth year students(compared than incoming students) had higher levels of resiliency in Action and Values, Independency and Determination and Self-confidence domains. Table 2 shows the correlations among academic stress, depressive symptoms, sleep quality, resiliency and quality of life in first and fourth year nursing students. Sao Paulo, 2016. 


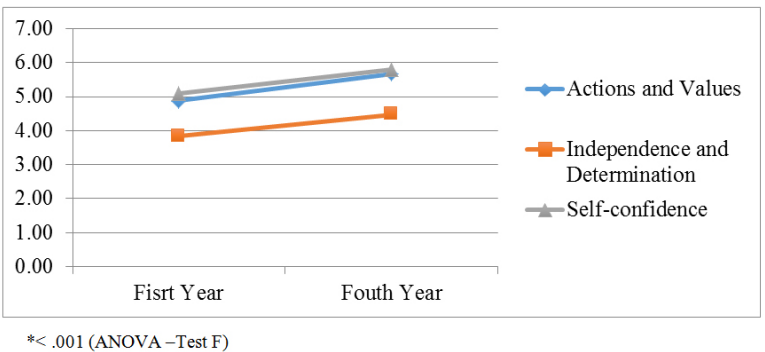

Figure 1. Comparison of means for each Resiliency Scale's domain in first and fourth year nursing students. Sao Paulo, 2016.
There was a statistical significant and positive correlation between stress and sleep quality. Once the PSQI scale is reverse, the higher levels of stress means poorer sleep quality. In addition, depressive symptoms was significant and negatively correlated with quality of life and resiliency, and positively correlated with sleep quality. It indicates that the relieving in depressive symptomatology is proportional to the improvement of sleep quality and quality of life. Also, higher resiliency levels may decrease depressive symptoms.

Table 2. Correlations among academic stress, depressive symptoms, sleep quality, resiliency and quality of life in first and fourth year nursing students. Sao Paulo, 2016

\begin{tabular}{llllll}
\hline & Stress & Depressive Symptoms & Sleep Quality & Quality of Life & Resiliency \\
\hline Stress & - & - & - & - & - \\
Depressive Symptoms & $\mathrm{R}=0.116$ & - & - & - & - \\
Sleep Quality & $\mathrm{R}=0.214^{*}$ & $\mathrm{R}=0.401^{*}$ & - & - & - \\
Quality of Life & $\mathrm{R}=-0.085$ & $\mathrm{R}=-0.506^{*}$ & $\mathrm{R}=-0.166$ & - & - \\
Resiliency & $\mathrm{R}=0.095$ & $\mathrm{R}=-0.385^{*}$ & $\mathrm{R}=-0.111$ & - & - \\
\hline
\end{tabular}

*Statistically significant correlation $(p<.05)$; ** Pearson Correlation Test.

\section{Discussion}

This study showed higher levels of academic stress in 4th grade students. In a survey of 1538 Chinese nursing students using Stress in Nursing Student scale, a higher level of stress was also observed among students in the last year of the course. ${ }^{[3]}$ In the last semesters of the course, the student experiences, in addition to the theoretical requirements, greater clinical workload, remaining longer in the clinical setting in situations that challenge their knowledge, attitudes and skills, ${ }^{[2]}$ being periods that require greater student responsibility and independence. ${ }^{[17]}$ In addition, close to the course ending and the consequent transition from student to nurse status, often students deal with uncertainties about theirs professional future. ${ }^{[18]}$ These characteristics-peculiar to the last semesters- may be more stressful to the nursing students.

In addition, there was no significant difference in sleep quality between the groups assessed, so sleep quality remains low (score $>5$ ) throughout the undergraduate course. Investigation performed with 372 medical students in an university of Sao Paulo interior demonstrated that $39.5 \%$ of sample had poor or very poor sleep quality, being that students from basic level of education reported the worst perception of subjective sleep quality and of daily disturbances than the others. ${ }^{[19]}$ It is possible that several curricular assignments, such as tests and homework, and extracurricular activities - including research and study groups, academic leagues, scholar directories - contribute in different ways across the academic

Published by Sciedu Press trajectory for the poor sleep quality of nursing students. However, in general, national and international ${ }^{[1,20]}$ studies have demonstrated that, independently of course semester, nursing students report poor sleep quality.

Although fourth years students had reported higher levels of stress and poorer sleep quality in all period assessed, these individuals showed lower intensity of depressive symptoms. In a study, where the Beck Depression Index was applied in 147 nursing students living in north of Brazil, 21.08\% of the sample reported moderate depressive symptoms and $3.40 \%$ severe symptoms of depression. After comparing different stages of nursing degree, the percentage of severe depression decreased from $6.98 \%$ in the first semester to $2.12 \%$ in the eighth semester. ${ }^{[21]}$ There are a few studies that compare depressive symptoms between different semesters of nursing undergraduate courses. However, it's possible that the higher levels of depressive symptoms in first year students be related with the changes in their lifestyle. In this period, students deal with the transition from high school to college and they start to experience a totally different routine and exigences specific from academic environment, what may require effort and adaptation, increasing the risk for the depressive moods. ${ }^{[18]}$ On other hand, insofar as the degree advances, students are more likely to get adapted to the academic context, getting to the last semester less vulnerable to the depression states. 
We found that fourth year students are more resilient than those starting the course, what indicated the strengthening of resilient features during the education process. This finding is in accordance with a qualitative research performed with third and fourth year nursing degree students from Singapore, in which students reported that, even stressed at the beginning of clinical classes, they become more resilient and able to adapt after accumulate several experiences in clinical and academic environments, being the classmates support an important factor in this process too. ${ }^{[22]}$ Face the importance of resiliency for coping with those challenges present in nursing students education, strategies for resiliency promotion need to be proposed and implemented for degree managers. ${ }^{[3,22]}$

Also, we observed that fourth year students have a better quality of life - in general and in the physical and psychological domains. This finding differs from those verified in investigation were the quality of life was lower in students at end of the course. ${ }^{[23]}$ In this sense, a research conducted with 630 health area students showed a lower quality of life in those enrolled in the last semesters. Researchers attribute these results to the increase in clinical activities at the final of the courses. However, this difference strength the statement of quality of life is a subjective phenomenon, depending on the individual's perception into their context of life and expectations. $^{[23]}$

In this sense, tiredness and lack of time for leisure, social and family activities may be elements that contribute to a poorer quality of life at beginning of the course in few contexts once it is the first contact with the academic setting and their demands. ${ }^{[22,23]}$ During the course, students may get adapted to several demands and learn to manage their tasks and academic responsibilities to have more time available for social and family convivence, what positively impact on the quality of life and strength their resilient personality. ${ }^{[22,23]}$

The increase in stress levels leads to student's poor sleep quality. This relation between high stress and poor sleep quality was also found in other investigation. ${ }^{[1,24]}$ These studies highlight the importance of supporting practices that relieve the stress levels, once is may improve the sleep quality, with potential benefits to academic performance and daily activities. ${ }^{[24]}$

Results also evidenced that the reduction in depressive symptoms scores lead to higher sleep quality and quality of life. This finding is similar to those from other studies, ${ }^{[24-26]}$ what

\section{REFERENCES}

[1] Benavente SBT, Silva RM, Higashi AB, et al. Influence of stress factors and socio-demographic characteristics on the sleep quality of demonstrates the relevance of supporting students in academic context trough interventions, such as pedagogic listening and relaxing practices. It may help students deal with anguish in daily life and reduce negative outcomes, such as depressive states and changes in sleep patterns, ${ }^{[26]}$ what compromises their social and familiar relationships, produces humor and behavior disturbances ${ }^{[21]}$ and reduces the academic performance. ${ }^{[21,26]}$

However, we observed that higher resilience leads to a reduction in depressive symptoms. Another study has shown that nursing students with less resilience were more likely to experience psychological adversities such as depression. ${ }^{[27]}$ Therefore, resiliency may be a strategy to deal with the academic issue that often lead to psychologic suffering, what rises the importance of resilience promotion programs.

\section{Conclusion}

Although the increase in stress levels from beginning to the end of the course, we verified that increase in levels of resiliency and quality of life, while depressive symptoms has decreased in period. So, we concluded that, even with the stressful experiences related to academic and personal life, there is a strengthening of resilient characteristics throughout the undergraduate course, which may explain the improvement in the students' quality of life. In this sense, stress, when effectively managed by individuals, can be a source of resilience development.

Nursing education environment has potential for disease and suffering, however, the experiences in this period seem to have a positive effect for developing resiliency in nursing students. Therefore, programs to promote resiliency need to be developed in academic setting since the begging of the courses in order to get a better general health and quality of life during the nursing education. Also, future investigation need to assess in greater depth the role of stress and individual features on resiliency development considering its protective effect on health.

As study limitations, we highlight the lack of longitudinal and cross-sectional studies comparing students' health status at beginning and ending of the course. It made the comparison between findings of this research to another ones involving students from the same area.

\section{CONFLiCTS OF INTEREST Disclosure}

The authors declare that there is no conflict of interest. nursing students. Rev Esc Enferm USP [Internet]. 2014 [cited 2018 Nov 10]; 48(3):514-20. PMid:25076281 https ://doi .org/10.1 $590 / \mathrm{S} 0080-623420140000300018$ 
[2] Costa ALS, Polak C. Construction and validation of an instrument for the assessment of stress among nursing students. Rev Esc Enferm USP [Internet]. 2009 [cited 2018 Nov 10]; 43(N.esp.): 1017-26. https://doi.org/10.1590/S0080-62342009000500005

[3] Smith GD, Yang F. Stress, resilience and psychological well-being in Chinese undergraduate nursing students. Nurse Educ Today. 2017; 49: 90-5. PMid:27889584 https://doi.org/10.1016/j.nedt .2016 .10 .004

[4] Chang SJ, Jang SJ. Social jetlag and quality of life among nursing students: A cross-sectional study. J Adv Nurs. 2018 Sep 19. [Epub ahead of print]

[5] Lazarus RS, Folkman S. Stress, appraisal, and coping. New York: Springer; 1984.

[6] Silva RM, Goulart CT, Lopes LFD, et al. Avaliação de estresse em estudantes de enfermagem - proposta de padronização da análise. In: Rossi AM, Meurs JA, Perrewé PL, (organizadores). Stress e qualidade de vida no trabalho: melhorando a saúde e bem-estar dos funcionários Porto Alegre: Atlas; 2013; 55-67.

[7] Filho NK, Teixeira MAP. A estrutura fatorial da Escala CES-D em estudantes universitários brasileiros. Aval psicol. [Internet]. 2011[citado 2018 out. 10]; 10(1): 91-7.

[8] Matos ACS, Oliveira IR. Terapia cognitivo-comportamental da depressão: relato de caso. Rev Ciênc Méd Biol. 2013; 12(n.spe): 512-9. https://doi.org/10.9771/cmbio.v12i4.9203

[9] Guo L, Deng J, He Y, et al. Prevalence and correlates of sleep disturbance and depressive symptoms among Chinese adolescents: a cross-sectional survey study. BMJ Open [Internet]. 2014[cited 2018 Nov 10]; 4(7): 1-9.

[10] Menon B, Karishma HP, Mamatha IV. Sleep quality and health complaints among nursing students. Ann Indian Acad Neurol. 2015; 18(3): 363-4. PMid:26425028 https://doi.org/10.4103/0972 $-2327.157252$

[11] The WHOQOL group. The World Health Organization Quality of Life assessment (WHOQOL): position paper from the World Health Organization. Soc Sci Med [Internet]. 1995 [cited 2018 Nov 10]; 41(10): 1403-9. https ://doi .org/10.1016/0277-9536(95)0 0112-K

[12] Taylor DJ, Bramoweth AD, Grieser EA, et al. Epidemiology of insomnia in college students: relationship with mental health, quality of life, and substance use difficulties. Behav Ther. 2013; 44(3): 339-48. PMid:23768662 https://doi.org/10.1016/j . beth. 2012.12 .001

[13] Wagnild GM, Young HM. Development and psychometric evaluation of resilience scale. J Nurs Meas [Internet]. 1993 [cited 2018 Nov 10]; 1: 165-78.

[14] Pesce RP, Assis SG, Avanci JQ, et al. Cross-cultural adaptation, reliability and validity of the resilience scale. Cad Saúde Pública. 2005; 21(2): 436-48

[15] Fleck MPA, Louzada S, Xavier M, et al. Application of the Portuguese version of the abbreviated instrument of quality life WHOQOL-bref. Rev Saúde Pública. 2000; 34(2): 17883. PMid:10881154 https://doi.org/10.1590/S0034-89102 000000200012

[16] Hair Jr JF, Anderson RE, Tatham RL, et al. Análise multivariada de dados. 5a. ed. Porto Alegre: Bookman; 2005.

[17] Cestari VR, Barbosa IV, Florêncio RS, et al. Stress in nursing students: study on sociodemographic and academic vulnerabilities. Acta Paul Enferm. 2017; 30(2): 190-6. https ://doi .org/10.1590/19 82-0194201700029

[18] Moreira DP, Furegato ARF. Stress and depression among students of the last semester in two nursing courses. Rev latinoam enferm. 2013; 21(Spec): [08 telas]. PMid:23459903 https://doi.org/10.159 0/S0104-11692013000700020

[19] Corrêa CC, Oliveira FK, Pizzamiglio DS, et al. Sleep quality in medical students: a comparison across the various phases of the medical course. J Bras Pneumol. 2017; 43(4): 285-9. PMid:29365004 https://doi.org/10.1590/s1806-37562016000000178

[20] Zhang Y, Peters A, Chen G. Perceived Stress Mediates the Associations between Sleep Quality and Symptoms of Anxiety and Depression among College Nursing Students. Int J Nurs Educ Scholarsh. 2018; 15(1): [9 telas]. PMid:29306924 https://doi.org/10.151 5/ijnes-2017-0020

[21] Trindade FTB, Gomes NN, Ferreira SEG, et al. Prevalência de Sintomas Depressivos em Acadêmicos de Enfermagem de uma Faculdade Privada em Belém-PÁ. Revista Científica Multidisciplinar Núcleo do Conhecimento [Internet]. 2017 [citado 2018 ago. 10]; 4: 24-38. https: //doi.org/10.32749/nucleodoconhecimento.com.br /saude/sintomas-depressivos-academicos-enfermagem

[22] Lopez V, Yobas P, Chow YL, et al. Does building resilience in undergraduate nursing students happen through clinical placements? A qualitative study. Nurse Educ Today. 2018; 67: 1-5. PMid:29704800 https://doi.org/10.1016/j.nedt.2018.04.020

[23] Paro CA, Bittencourt ZZLC. Qualidade de Vida de Graduandos da Área da Saúde. Rev bras educ méd. 2013; 37 (3): 365-75. https://doi.org/10.1590/S0100-55022013000300009

[24] Lemma S, Gelaye B, Berhane Y, et al. Sleep quality and its psychological correlates among university students in Ethiopia: a crosssectional study. BMC Psychiatry. 2012; 12: 237. PMid:23270533 https://doi .org/10.1186/1471-244X-12-237

[25] Trockel M, Manber R, Chang V, et al. An e-mail delivered CBT for sleep-health program for college students: effects on sleep quality and depression symptoms. J Clin Sleep Med. 2011; 7(3): 276-81.

[26] Souza IMDM, Paro HBMS, Morales RR, et al. Health-related quality of life and depressive symptoms in undergraduate nursing students. Rev latinoam enferm. [Internet]. 2012 Aug [cited 2018 Dec 06]; 20(4): 736-43. PMid:22990159 https://doi .org/10.1590/S0 104-11692012000400014

[27] Reeve K, Shumaker C, Yearwood E, et al. Perceived stress and social support in undergraduate nursing students' educational experiences. Nurse Educ Today. 2013; 33: 419-24. PMid:23246284 https://doi.org/10.1016/j.nedt.2012.11.009 